\title{
The newsvendor problem with advertising: an overview with extensions
}

\author{
Dušan Hrabec • Kjetil K. Haugen • \\ Pavel Popela
}

Received: date / Accepted: date

\begin{abstract}
The effect of advertising on sales has been the subject of recent studies as an important aspect in many demand-based problems. Herein, we deal with the newsvendor problem, due to its simple structure, as a suitable tool for illustrating how facets of marketing may affect decision-making concerning operational problems. In the setting presented, the newsvendor is faced with advertising-sensitive stochastic demand, where a demand-related random element comprises an advertising decision of the multiplicative or additive form. We assume that a suitable advertising strategy results in increased sales. Two advertising response functions are considered, these being concave downward and S-shaped. We review and extend the existing results relating to the newsvendor problem with marketing effects, which mostly pertain to the concave function. These are generalized by defining the S-shaped function, and some original insights into the effect of advertising are given. We establish that the optimal advertising expenditure for the multiplicative case is always less than or equal to the optimal amount in the equivalent deterministic model while it is always equal in the additive case. We finally illustrate the results that are obtained by providing numerical examples involving various advertising response functions, as well as management-related interpretations.
\end{abstract}

D. Hrabec*

Tomas Bata University in Zlín, Faculty of Applied Informatics, Nad Stráněmi 4511, 76005 Zlín, Czech Republic

* E-mail: hrabec@fai.utb.cz and hrabec.dusan@gmail.com

K.K. Haugen

Molde University College - Specialized University in Logistics, Britvegen 2, 6402 Molde, Norway

D. Hrabec and P. Popela

Brno University of Technology, Faculty of Mechanical Engineering, Institute of Mathematics, Technická 2896/2, 61669 Brno, Czech Republic 
Keywords Newsvendor problem with advertising · Marketing and operation interface $\cdot$ Advertising response function - Stochastic optimization · Decisionmaking

\section{Introduction}

Coordinating marketing and production decisions still ranks as one of the most challenging practical and theoretical problems of operations management. Indeed, a number of producers have used innovative marketing strategies to gain effective control of their inventories. This paper discusses the problem of simultaneously determining the quantity necessary to order and the advertising expenditure required for a product for which demand is random.

We approach the problem by formulating a stochastic single-period problem, commonly referred to as the newsvendor problem (NP). A typical NP reveals that the quantity ordered maximizes the expected profit. In the setting given herein, the newsvendor is faced with advertising-sensitive stochastic demand where the demand-related random element depends on advertising decisions. We consider that a suitable advertising strategy can lead to increases in sales. Note that the assumption of a fixed price corresponds to an instance of the buyers effectively representing mere price-takers.

Although, NP has been studied for decades, it still serves as a suitable tool to illustrate many new marketing situations (Dai and Meng 2015; Jammernegg and Kischka 2007; Shi and Zhang 2014; Xinsheng et al 2015). In this paper, we use notation that is frequently utilized for the newsvendor problem with pricing (NPP) in order to show how marketing aspects interact with production decisions in the newsvendor problem with advertising (NPA). We follow up on our earlier modeling ideas, as presented in Hrabec et al (2012), in which some results from NPP were reviewed and illustrated. Moreover, inspired by Petruzzi and Dada (1999), who presented findings on NPP for the linear price-demand function in an additive demand model, as well as the hyperbolic price-demand function for a multiplicative demand case (higher prices cause a decrease in demand), we present suitable (and more complex) functions related to various advertising situations for additive and multiplicative cases (an increase in advertising expenditure brings about higher sales). Using suitable notations and procedures, it is our aim to further the understanding of the matter and to present new results.

\subsection{Literature review}

The various sources of literature pertaining to the subject are detailed below. Prior to introducing the model as one comprising a combination of conceptual notions, a review is given on the primary resources utilized. 


\subsubsection{The newsvendor problem}

The literature on NP, which has a long and interesting history, was instigated in 1888 by Edgeworth (1888), who developed the concept to tackle a bank cash-flow problem. As previously mentioned, NP is based upon the notion of discerning the quantity of a particular item to be ordered but with no insight into future circumstances. The objective of this stochastic single-period problem is to determine said quantity for a fixed period of time maximizing expected total profit. Porteus (1990) and Khouja (1999) present useful reviews for conducting a basic static case study and its generalizations. Examples of NP serving to illustrate the general principles of mathematical and stochastic programming are provided by Dupačová (1994), Ruszczynski and Shapiro (2003) and Shapiro et al (2009). The simple structure of NP, makes it an ideal tool for examining the interaction of operational and marketing issues and the resultant impact on a decision-making process (Petruzzi and Dada 1999).

\subsubsection{The newsvendor problem with pricing}

The first mathematical formulation for price effects associated with inventory control problems was presented by Whitin (1955). Prior to this, economic theoreticians had disregarded a number of important aspects. Hence, Whitin decided to focus on such overlooked aspects with special emphasis on the demand. He adapted the NP model, modifying it so the unit selling price became a decision variable and demand linearly depended on the selling price per unit under conditions of a retailer knowing the probability distribution of demand. Consequently, the retailer was aware of the amount required to meet the demand at any given price. Whitin established a sequential method for first determining the optimal quantity for the order as a function of the selling price, followed by the relevant optimal price. Mills (1959) extended the NPP model by specifying the mean demand as a function of the selling price. He refined Whitin's methodology by modeling uncertainty in the additive form, the demand being specified as $D(p, \xi)=d(p)+\xi$ where $d(p)$ is a decreasing demand function of price $p$, and $\xi$ is a random variable defined in a certain domain. Specifically, Mills established that an optimal price under uncertain demand is never greater than the optimal price set by equivalent deterministic monopoly models; this is known as the riskless price. Both Whitin and Mills considered the single period form of the model, i.e., a static problem, where only a single price and the quantity to be ordered are the decision variables. Detailed comparison between static and dynamic cases is provided by Karlin and Carr (1962). To both, they applied the concept of additive demand, as well as a case of multiplicative demand; the latter was defined as $D(p, \xi)=d(p) \xi$. Regarding the dynamic model (the multi-period problem), they employed infinite-period approximation to the $n$-period model. However, they did so assuming that it was necessary to determine a single constant price at the commencement of the planning horizon. They established that an optimal price under multiplicative 
uncertain demand is never less than the riskless price, which is an outcome opposing that arrived at by Mills for additive uncertainty.

For overviews of NPP and other recent results, see Petruzzi and Dada (1999) and Yao et al (2006). See Monahan et al (2002) for its dynamic case.

\subsubsection{Advertising}

Advertising policy is a crucial aspect of marketing. The key questions are the following: What is the effect for the retailer? How much should be spent on advertising? How often? What marketing channel should be used? The authors Jones (1995) and Bronnenberg (1998) published two possible approaches to expenditure frequency - a constant advertising stream (extremely expensive) or pulses of advertising conducted at irregular intervals (the preferred option); see also Khouja and Robbins (2003). A major issue is that concerning the actual effectiveness of advertising. Advertising response functions are typically assumed to adhere to the following: (1) a concave-downward function (usually an advertising response function without a threshold); (2) an S-shaped logistic function (an advertising response function with a threshold); for reference purposes see Bass (1969), Simon and Arndt (1980), Bagozzi (1986), Jones (1995) and Lee and Hsu (2011). In general, the responsiveness of sales to advertising may start to decline beyond a level of spending on advertising (Batra et al 1995). This phenomenon of diminishing returns is better represented by a downward-sloping curve on a graph in which the advertising levels relate to the sales results. Alternatively, some modelers prefer to assume that the relationship is actually S-shaped. Initially, when advertising budgets are low, sales exhibit no response to advertising at all. It is supposed that it takes some time for advertising to take effect as repeated exposure is required. Afterwards, a point of increasing returns is discerned when sales really begin to respond to further advertising once the advertising budget exceeds a minimum, critical-level threshold. Eventually, the curve begins to slope downwards again, when the phase of diminishing returns reappears; see also Hanssens and Parsons (1993) for further analysis of the S-shaped function. Publications by Phillips (2005), Mooradian et al (2011) and Kotler and Armstrong (2013) provide a comprehensive overview on such themes and proceed to detail recent principles of marketing.

\subsubsection{The newsvendor problem with advertising}

A single period inventory problem with advertising was given by Gerchak and Parlar (1987). Furthermore, the interface between decision-making on marketing and manufacturing has been studied by many researchers, examples being Eliashberg and Steinberg (1993), Celikbas et al (1999), Malhotra and Sharma (2002), Hausman et al (2002), Freeland (1980) and Leitch (1974), to whom reference is made in this paper.

The effect exhibited by advertising on sales represents an important aspect of demand-based problems. When a retailer/newsvendor faces demands of the 
stochastic advertising-sensitive type, he is forced to make decisions concerning advertising and inventory prior to the demand being met (Wang 2011). Khouja and Robbins (2003) and Wang (2011) assume that the mean demand is strictly increasing and strictly concave in advertising expenditure. Khouja and Robbins (2003) provide an extensive review of related literature and the advertising problem, attempting to solve the problem for uniform, exponential and normal demand distributions. Wang (2008) investigated circumstances under which advertising leads to increased sales under additive demand. Later, Wang (2009) extended the problem to cover the situation pertaining to a perishable product subject to being ordered under emergency conditions, first for a multiplicative model, then going on to investigate the general demand function in Wang (2011). Finally, Wang and Hu (2011) applied the results to coordinating a supply chain with advertising, as well as setting a price for the manufacturer.

\subsection{Outline of the paper}

Section 2 commences with outlining the impetus for the study. Afterwards, the advertising response function is introduced, the demand function and the related random impact are stated and the underlying NPA is described. Section 3 describes the objective function being rewritten to suit the multiplicative demand model. Then, optimal stocking quantity, which corresponds to a standard NP result, is analyzed and crucial assumptions and theorems are provided. A comparison is made between the optimal advertising amount obtained and the result of the riskless problem. Section 4 introduces the NPA model in the additive form, and summarizes the general modeling differences and advertising results as related to the multiplicative demand case. Additionally, numerical results are provided for the uniform distribution of the random variable in the multiplicative case for the NPA model as well as numerical figures for the equivalent NP (section 5). Finally, the paper concludes with section 6 , which also features managerial interpretation. Remarks on applicability of results and further research possibilities follow.

\section{Advertising response function and the newsvendor problem}

Modern goods and service production markets are characterized by increased competition. Globalization and focus on increased global trade drives this development. One obvious consequence is increased demand uncertainty. At the same time, marketing strategies (locally and globally) are a necessity in order to sell almost any product. This combination - increased demand uncertainty and marketing necessity - indicates that formal modeling spawning both dimensions holds managerial importance. A simple newsvendor model platform may prove to be a relevant and principal "laboratory" for increased understanding on how management science may be applied in order to solve these problems. 
One of the keys to understanding the marketing problems lies in the relation between demand and advertising response function. Therefore, we first focus on the response function. Then, the basic concepts of the demand function follow together with the definition of its related uncertainty. Finally, we combine the above mentioned aspects from perspective of a company (e.g. a retailer) sourcing a product with short life cycle to stock using the framework of the renowned newsvendor model, which will serve as a suitable tool for illustrating how marketing aspects may affect decision making in demand-based (operational-marketing) problems.

\subsection{Advertising response function}

The response function describes the sales effect of additional amounts of advertising, even though it sometimes illustrates the amount of advertising needed to trigger buying (Jones 1995). We further use two (general) functions that are often used: a) the concave response function, which is presented in subsection 2.1.1 (Jones 1995; Khouja and Robbins 2003); b) the S-shaped response function, which is presented in subsection 2.1.2 (Jones 1995).

Although the S-shaped function is very important from the marketing literature perspective, it has not yet been considered by researchers dealing with operational research in the discussed context. According to the marketing literature trying to approximate the advertising situations, we define the S-shaped function as a bounded real function defined for all nonnegative input values with a positive derivative at each point, which is first convex and then concave. It means, in the beginning, when advertising budgets are low, sales do not respond significantly to advertising. It supposedly takes time for advertising wear-in. We see the point of increasing returns, as sales really begin to respond to increased advertising, as the advertising budget exceeds some minimum critical-level threshold. Finally, the curve begins to slope downward again, as once again the diminishing-returns phase appears, see Bass (1969) and Bagozzi (1986).

Let the response function $d(a)$ be continuous, nonnegative, twice-differentiable and increasing on its domain $\left[0, a_{\max }\right]$ in the advertising expenditure $a$ (Khouja 1999; Lee and Hsu 2011). Moreover, since $d(0)>0, d(a)$ is positive.

To capture a real situation/dependency between the advertising expenditure and demand, we further present three particular functions, which we provide for their illustrative-suitable behaviour.

\subsubsection{Suitable examples of the concave response function}

Advertising function without threshold in demand. Here, we consider an advertising function with diminishing returns, which is given by

$$
d(a)=d_{0}+\omega a^{\alpha},
$$


where $\alpha \in[0,1]$ and $\omega>0$ are empirically determined constants indicating the effectiveness of advertising and $d_{0}>0$ represents the initial demand (for $a=0$ ). If $\omega=0$, then the demand is independent of advertising expenditure. The larger the value of $\alpha$, the more effective advertising is. For more details on the function and its analysis, see Khouja and Robbins (2003).

Advertising function with threshold in demand. Here, the function $d(a)$ has an asymptotic behavior (an upper bound in demand). Based on the empirical study by Dubé et al (2005), we suggest the following function as a suitable candidate:

$$
d(a)=d_{0}+\theta\left[1-\frac{1}{(a+1)^{\delta}}\right]
$$

where $\theta$ and $\delta$ are positive real numbers and $d_{0}$ is an initial demand. This function has a horizontal asymptote defined by demand value $\theta+d_{0}$. The choice of $\delta$ decides the speed towards the asymptote. A small value of $\delta$ indicates a slow speed while a large value of $\delta$ indicates a fast speed.

\subsubsection{S-shaped response function example}

A logistic function/curve represents a typical "S" shape graph. According to theoretical definitions of the S-shaped response function (Bass 1969; Simon and Arndt 1980; Bagozzi 1986; Jones 1995; Lee and Hsu 2011) and according to the experimental marketing research results (e.g. Dubé et al 2005), we suggest

the following function:

$$
d(a)=d_{0}+\frac{\theta}{1+\left(\frac{\theta-\theta_{l}}{\theta_{l}}\right) e^{-\gamma a}},
$$

where $\theta$ specifies an upper asymptote, $\gamma$ is a coefficient of growth, $\theta_{l}$ defines a lower asymptote (see, e.g., Fox and Weisberg 2010 for a mathematical description/analysis of the function). We also refer to other Sshaped functions as the Gompertz function.

Figure 1 illustrates three particular examples of the above mentioned functions. Section 3.2.1 provides numerical examples involving these functions.

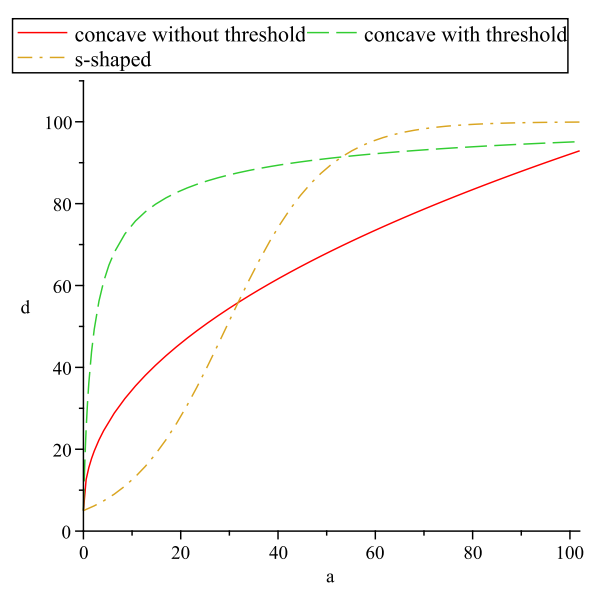

Fig. 1: Advertising response functions $d(a)$. 


\subsection{Demand function and randomness}

However, from the managerial perspective, we deal with problems where the decision-maker does not know the real demand. Therefore, we further model the demand as a (response) function, which can be affected by the advertising expenditure and which somehow depends on a random element.

Inspired by many papers on the NPP (Mills 1959; Karlin and Carr 1962; Petruzzi and Dada 1999; Yao et al 2006) and some on the NPA (Khouja 1999; Wang 2008, 2011), we further assume that advertising-related randomness is independent of the demand, which helps us avoid complexities.

Let us denote the demand function $D\left(a, \xi_{a}, \xi_{m}\right)$ and let it satisfy

$$
D\left(a, \xi_{a}, \xi_{m}\right)=d(a) \xi_{m}+\xi_{a},
$$

where $\xi_{a}, \xi_{m}$ are independent continuous random variables. In the study, we will further deal with two special cases of demand function $D\left(a, \xi_{a}, \xi_{m}\right)$ : a) the multiplicative demand case (section 3 ) and b) the additive demand case (section 4$)$. In order to define the multiplicative demand case, let $\mathrm{P}\left(\xi_{a}=0\right)=1$ and let the random variable $\xi_{m}$ be defined on the domain $\left[A_{m}, B_{m}\right]$ and satisfy $E\left[\xi_{m}\right]=1$. In the additive demand case, let $P\left(\xi_{m}=1\right)=1$ and let the random variable $\xi_{a}$ be defined on the domain $\left[A_{a}, B_{a}\right]$ and satisfy $E\left[\xi_{a}\right]=0$. Then, for both cases, the expectation of $D$ is specified as: $E\left[D\left(a, \xi_{a}, \xi_{m}\right)\right]=d(a)$.

In comparison with the NPP references where the additive form of the randomness is commonly used for the linear demand function while the multiplicative form for the hyperbolic demand function, we examine the effects of the additive as well as multiplicative form on the optimal advertising strategy for the concave and the S-shaped functions, which also corresponds to ideas in the NPP study (Yao et al 2006).

Before we model and examine the multiplicative demand form in section 3 and the the additive demand form in section 4 , we introduce the NPA formulation that provides us with a suitable aid for the study.

\subsection{The newsvendor problem with advertising}

Let us assume the following situation: First, we decide about an amount $a$ to advertise for a product to be sold and we simultaneously buy and stock $x$ units of the product for a unit cost $c$. Then, the selling period begins. If demand $D$ is greater than $x$, all stocked units are sold for revenue $p x$, where $p$ is a unit price, $p>c$. In this case, we consider a loss given by a unit shortage penalty cost $s$ for all shortages, $D-x$. Otherwise, if demand $D$ is less or equal to $x$, the revenue is only $p D$ and leftovers, $x-D$, are salvaged through a unit salvage value $v, v<c$. Then, the objective (profit) function is denoted by $\pi\left(a, x, \xi_{m}, \xi_{a}\right)$ being defined as follows:

$$
\pi\left(a, x, \xi_{a}, \xi_{m}\right)= \begin{cases}p x-c x-s\left[D\left(a, \xi_{a}, \xi_{m}\right)-x\right]-a, & x<D, \\ p D\left(a, \xi_{a}, \xi_{m}\right)-c x+v\left[x-D\left(a, \xi_{a}, \xi_{m}\right)\right]-a, & x \geq D .\end{cases}
$$


The decision variables are the order quantity denoted by $x$ and the amount spent on the advertising denoted by $a$, while the demand $D\left(a, \xi_{a}, \xi_{m}\right)$, which depends on the advertising expenditure $a$ and is affected by the random elements $\xi_{a}, \xi_{m}$, is not completely known when the decisions are made.

\section{Multiplicative demand model}

Let the demand function $D\left(a, \xi_{a}, \xi_{m}\right)$ be defined in the multiplicative form (see subsection 2.2) and let $F_{m}(\cdot)$ denote a cumulative distribution function (cdf) and $F_{m}(\cdot)$ be a probability density function (pdf) of $\xi_{m}$. In order to assure that demand is positive, we require that $A_{m}>0$. Then, the demand is in the multiplicative form

$$
D_{M}\left(a, \xi_{m}\right)=d(a) \xi_{m}
$$

see Karlin and Carr (1962) for similar ideas in the NPP. The objective function (5) can be rewritten by substituting (6) and utilizing the 'stocking factor' defined as

$$
z=\frac{x}{d(a)}
$$

where $z \geq 0$ (note that if $x=0$ and since $d(a)>0$, then $z=0$ ). A similar transformation of the objective (variable transformation, respectively) has already been used to simplify the calculations in the NPP (Petruzzi and Dada 1999; Zhan and Shen 2005). It provides an alternative interpretation of the stocking decision: if the choice of $z$ is greater than the realized value of random variable $\xi_{m}$, then leftovers occur, otherwise shortages occur (Petruzzi and Dada 1999). An important managerial interpretation for $z$ demonstrates that, although $z$ is defined differently for each of the two mentioned demand cases (see section 4 for the additive case), its meaning is consistent for both: $z$ represents a stocking factor that Petruzzi and Dada (1999) defined as a surrogate for safety factor by Silver and Peterson (1985).

Then, we obtain:

$$
\pi\left(a, z, \xi_{m}\right)= \begin{cases}p z d(a)-c z d(a)-s d(a)\left[\xi_{m}-z\right]-a, & \text { for } z<\xi_{m} \\ p \xi_{m} d(a)-c z d(a)+v d(a)\left[z-\xi_{m}\right]-a, & \text { for } z \geq \xi_{m}\end{cases}
$$

For better understanding we unify notation for both multiplicative and additive cases and the same symbols $\pi$ are also used for both objective functions involving either $x$ or $z$ variables.

The objective is to maximize the expected profit by choosing $a$ and $z$. However, the optimal solution is not necessarily an interior solution, in particular, the value of $z$ can be on the boundary, $A_{m}$ or $B_{m}$ (Zhan and Shen 2005). 
We can express the expected profit $\Pi(a, z)$ as:

$$
\begin{aligned}
\Pi(a, z)= & E\left[\pi\left(a, z, \xi_{m}\right)\right]=d(a) \int_{A_{m}}^{z}[p t+v(z-t)] F_{m}(t) d t \\
& +d(a) \int_{z}^{B_{m}}[p z-s(t-z)] F_{m}(t) d t-c z d(a)-a
\end{aligned}
$$

Defining the riskless profit (Mills 1959; Petruzzi and Dada 1999), which would occur in the absence of uncertainty, as

$$
\Psi(a)=(p-c) d(a)-a,
$$

and the so-called expected loss per unit as

$$
l(z)=(c-v) \Lambda(z)+(p+s-c) \Theta(z)
$$

where $d(a) \Lambda(z)$ denotes expected leftovers and $d(a) \Theta(z)$ expected shortages, the expected profit given by $(8)$ can be rewritten as

$$
\Pi(a, z)=\Psi(a)-L(a, z)=d(a)[p-c-l(z)]-a .
$$

Note that $L(a, z)=d(a) l(z)$ is the expected loss that occurs as a result of the presence of uncertainty (Petruzzi and Dada 1999; Silver and Peterson 1985) and $p-c-l(z)$ denotes the so-called per-unit expected benefit, i.e., margin minus expected loss. If $z$ is chosen too high, in (11) or in (10), respectively, an overage cost $(c-v)$ appraises each of the $d(a) \Lambda(z)$ expected leftovers, and, if $z$ is chosen too low, an underage cost $(p+s-c)$ appraises each of the $d(a) \Theta(z)$ expected shortages. The equivalence of expressions (8) and (11) can be obtained by a sequence of straightforward substitutions.

Remark 1 The transformation from $x$ to $z$ presents an advantage of determining optimal values $a^{*}$ and $x^{*}$ that maximize the expected profit, using the following steps:

1. We firstly determine an optimal stocking factor $z^{*}$ using input parameters (subsection 3.1). Because of the form of (11) and (8) we can obtain $z^{*}$ independently on optimal $a$ value.

2. Using a suitable function $d(a)$, we can express an optimal advertising $a^{*}$ and we determine an optimal order quantity $x^{*}$ such that $x^{*}=z^{*} d\left(a^{*}\right)$ (subsection 3.2).

\subsection{Optimal stocking quantity}

To maximize $\Pi(a, z)$ over two variables, we follow two steps described in Remark 1. Solving the first order condition (with respect to $z$ ), we get an expression for optimal $z$ :

$$
F_{m}\left(z^{*}\right)=\frac{p+s-c}{p+s-v} .
$$


It is comparatively easy to show that $\Pi(a, z)$ is concave in $z$ on $[0, \infty)$ : $\frac{\partial^{2} \Pi(a, z)}{\partial z^{2}}=(v-p-s) F_{m}(z) d(a)$, where $v-p-s<0$. Moreover, assuming that $F_{m}$ is invertible, we can express the optimal and unique $z^{*}$ as

$$
z^{*}=F_{m}^{-1}\left(\frac{p+s-c}{p+s-v}\right)
$$

which corresponds to the standard NP result (Whitin 1955; Porteus 1990).

\subsection{Optimal advertising expenditure}

Substituting (12) into (11), we get the following expression:

$$
\Pi\left(a, z^{*}\right)=d(a)\left[p-c-l\left(z^{*}\right)\right]-a,
$$

where $l(z)$ is given by (10). Notice in (13) that, if $p-c-l\left(z^{*}\right)<0$, the expected profit $\Pi\left(a, z^{*}\right)$ is, under our assumptions about $d(a)$ in subsection 2.2, negative and strictly decreasing in $a$, which does not capture any real situation (Wang 2011), similarly if $p-c-l\left(z^{*}\right)=0$. So, we get the following assumption.

Assumption 1 The per-unit expected benefit must be positive, i.e., $p-c-$ $l\left(z^{*}\right)>0$.

This assumption simply means that the expected (average) profit per unit is greater than zero (i.e., price $p$ minus cost $c$ minus expected loss per unit $l\left(z^{*}\right)$ is greater than zero). Otherwise, if we expect a loss, the only good strategy is to "do nothing" ( $x=0, a=0$ and so $z=0)$, see section 5 for illustrative examples. As Assumption 1 depends on the expected loss function $l(z)$, it also depends on the distribution $F_{m}$. The more shortages or leftovers we expect (caused, for example, by greater variance of the distribution), the greater is the expected loss $l(z)$. Similarly to other parameters from the expected loss function given by (10), e.g., as $s$ increases, so does $l(z)$. Since the assumption is crucial for further analysis, we provide more detailed insights for the uniform distribution in subsection 5.1.

Let us assume the expected profit expression given by (13). Solving the first order condition of $\Pi\left(a, z^{*}\right)$ with respect to $a$, we get the following remark.

Remark 2 The optimal advertising expenditure $a^{*}$ must satisfy the (necessary) optimality condition, which is given by:

$$
\frac{\mathrm{d} d(a)}{\mathrm{d} a}=\frac{1}{p-c-l\left(z^{*}\right)} .
$$

\subsubsection{Optima for advertising response function examples}

Note that the following expressions for optimal advertising expenditure are true for the multiplicative demand case, while, for the additive demand case, we must substitute $l\left(z^{*}\right) \equiv 0$ to obtain the results, see/compare (14) and (16).

The following examples/expressions are computed for advertising response functions given by (1) and (2), that are concave functions, and (3), that is S-shaped function. 
1. Advertising function without threshold in demand. Solving the first order condition for $\Pi\left(a, z^{*}\right)$ with respect to $a$, we get

$$
a^{*}=\sqrt[1-\alpha]{\omega \alpha\left[p-c-l\left(z^{*}\right)\right]}
$$

2. Advertising function with threshold in demand. Similarly, we get here

$$
a^{*}=\sqrt[\delta+1]{\theta \delta\left[p-c-l\left(z^{*}\right)\right]}-1
$$

3. S-shaped response function example. Here, the optimal advertising for the $\mathrm{S}$-shaped function is expressed by:

$$
a^{*}=-\frac{\ln \left(\frac{\left\{1-\frac{1}{2}\left[p-c-l\left(z^{*}\right)\right] \theta \gamma+\frac{1}{2} \sqrt{-4\left[p-c-l\left(z^{*}\right)\right] \theta \gamma+\left[p-c-l\left(z^{*}\right)\right]^{2} \theta^{2} \gamma^{2}}\right\} \theta_{l}}{-\theta+\theta_{l}}\right)}{\gamma} .
$$

\subsubsection{Monotonocity}

Let us take the second derivative of the expected profit given by (13). We get

$$
\frac{\mathrm{d}^{2} \Pi\left(a, z^{*}\right)}{\mathrm{d} a^{2}}=\frac{\mathrm{d}^{2} d(a)}{\mathrm{d} a^{2}}\left[\left(p-c-l\left(z^{*}\right)\right]\right.
$$

Then, due to Assumption 1, the following lemma is obtained from (15).

Lemma 1 The intervals of concavity and convexity of the expected profit $\Pi\left(a, z^{*}\right)$ with respect to a are identical with the intervals of concavity and convexity of the response function $d(a)$.

The following assumption, together with Assumption 1, will further help us to guarantee solution/optimality uniqueness for selected types of demand functions (i.e. for the concave and the S-shaped function). The assumption results from expression (11), or (14) respectively.

Assumption 2 The demand function d(a) satisfies that $\lim _{\Delta a \rightarrow 0_{+}} \frac{d(\Delta a)-d(0)}{\Delta a}>$ $\frac{1}{p-c-l\left(z^{*}\right)}$ and $\lim _{\Delta a \rightarrow 0_{+}} \frac{d\left(a_{\max }\right)-d\left(a_{\max }-\Delta a\right)}{\Delta a}<\frac{1}{p-c-l\left(z^{*}\right)}$.

Remark 3 In such case, where the function $d(a)$ is defined on a higher range than $\left[a, a_{\text {max }}\right]$, the conditions can be rewritten to: $\frac{\mathrm{d} d(0)}{\mathrm{d} a}>\frac{1}{p-c-l\left(z^{*}\right)}$ and $\frac{\mathrm{d} d\left(a_{\max }\right)}{\mathrm{d} a}<\frac{1}{p-c-l\left(z^{*}\right)}$. 


\subsubsection{Concave response function}

Suppose that the demand/response function $d(a)$ is strictly concave in domain of $a$ (Khouja and Robbins 2003), see Figure 1. Then, we can deduce the following theorem.

Theorem 1 If the response function $d(a)$ is strictly concave, then, under assumptions 1 and 2, the expected profit $\Pi\left(a, z^{*}\right)$ is strictly concave in a and so the globally optimal advertising expenditure $a^{*}$ is unique and is given by solution of (14) with respect to decision variable $a$.

Proof Since the response function $d(a)$ is considered to be strictly concave in its domain then under Assumption 1 and Lemma 1, we obtain that $\Pi\left(a, z^{*}\right)$ is also strictly concave in $a$, see (15). Moreover, under Assumption 2, we know the expected profit $\Pi\left(a, z^{*}\right)$ to be increasing at the initial point and decreasing at the end point. Then, the critical point determined from the optimality condition (14) is unique and is the optimal advertising amount $a^{*}$ (see section 5 for illustrative examples).

\subsubsection{S-shaped response function}

Theorem 2 If the response function $d(a)$ is $S$-shaped, then, under assumptions 1 and 2, the expected profit $\Pi\left(a, z^{*}\right)$ is strictly quasi-concave in a and so the globally optimal advertising expenditure is unique and is given by (14).

Proof Since the response function is supposed to be S-shaped, under Assumption 1 , we know that the expected profit function $\Pi\left(a, z^{*}\right)$ is also first convex and then concave in $a$. Moreover, using Assumption 2, the expected profit $\Pi\left(a, z^{*}\right)$ increases at the initial point and so it will increase until it reaches its maximum. In other words, $\Pi\left(a, z^{*}\right)$ is strictly quasi-concave in $a$. Then, from the optimality condition (14), we can get one critical point $a^{*}$ presenting the optimal advertising amount, which always lies in the concave range (see section 5 where we provide one illustrative and one counter example).

In order to solve the original problem of maximizing the expected value of the objective function given by (5) with respect to decision variable $x$, a final step is to determine an optimal order quantity $x^{*}$ from $(7)$. The pair $\left[a^{*}, x^{*}\right]$ then presents the optimal solution of the original NPA given by (5) for the multiplicative demand case defined by (6) for the expected objective function case, see (8) and (11).

\subsubsection{Comparison with riskless problem}

Consider the advertising decision without demand uncertainty and note that the profit of such a deterministic problem is called riskless profit, $\Psi(a)$, given by (9). Solving the first order condition of $\Psi(a)$, we get the following necessary optimality condition: 


$$
\frac{\mathrm{d} d(a)}{\mathrm{d} a}=\frac{1}{p-c}
$$

which must be satisfied by the optimal riskless advertising $a_{\Psi}^{*}$.

Remark 4 If the response function $d(a)$ is either concave or S-shaped, then, under Assumption 2, the necessary optimal condition (16) is also sufficient for the optimal riskless advertising $a_{\Psi}^{*}$ as we can adequately apply (15) and Lemma 1.

Based on the optimality condition (16), we can prove the following theorem under assumptions 1 and 2 considering concave and S-shaped functions.

Theorem 3 For the multiplicative demand model, the optimal advertising $a^{*}$ is always less than or equal to the optimal riskless advertising $a_{\Psi}^{*}$.

Proof Using expressions (14) and (16), we know that $\frac{1}{p-c} \leq \frac{1}{p-c-l\left(z^{*}\right)} \Rightarrow$ $\frac{\mathrm{d} d\left(a_{\Psi}^{*}\right)}{\mathrm{d} a} \leq \frac{\mathrm{d} d\left(a^{*}\right)}{\mathrm{d} a}$. For both functions, concave and S-shaped, the optimal advertising $a^{*}$, if it exists and is greater than zero, belongs to the concave part of $d(a)$. Then, for the concave part of $d(a)$, we know that $\frac{\mathrm{d} d(a)}{\mathrm{d} a}$ is decreasing and so if $\frac{\mathrm{d} d\left(a_{\Psi}^{*}\right)}{\mathrm{d} a} \leq \frac{\mathrm{d} d\left(a^{*}\right)}{\mathrm{d} a}$ then $a_{\Psi}^{*} \geq a^{*}$. See section 5 for illustrative examples.

Remark 5 Recall that the optimal price for multiplicative uncertain demand is not less than the riskless price in the NPP (Petruzzi and Dada 1999).

Even though there are similar structures of the the expected profit functions, which is $\Pi(p, z)=\Psi(p)-L(z, p)$ in the NPP case, while the NPA equivalent is given by (11), the demand function is defined differently: $d(p)$ is decreasing in $p$ in the pricing case, but $d(a)$ increases in $a$ in the advertising case. Therefore, it is not surprising that the observation on the effect of uncertainty given by Theorem 3 is opposite to its NPP equivalent (see Remark 5).

\section{Additive demand model}

Let the demand function $D\left(a, \xi_{m}, \xi_{a}\right)$ be defined in the additive form (see subsection 2.2) and let $F_{a}(\cdot)$ denote the cdf and $F_{a}(\cdot)$ the pdf of $\xi_{a}$. Then, the demand is in the additive form

$$
D_{A}\left(a, \xi_{a}\right)=d(a)+\xi_{a} .
$$

Considering model (5), demand function (17) and defining the stocking factor $z \in \mathbb{R}$ as $z=x-d(a)$ (if $x=0$ then $a=0$ but $d(0)>0$ ), we get:

$$
\pi\left(a, z, \xi_{a}\right)= \begin{cases}p[d(a)+z]-c[d(a)+z]-s\left[\xi_{a}-z\right]-a, & \text { for } z<\xi_{a} \\ p\left[d(a)+\xi_{a}\right]-c[d(a)+z]+v\left[z-\xi_{a}\right]-a, & \text { for } z \geq \xi_{a} .\end{cases}
$$

The expected profit $\Pi(a, z)$ can be expressed by:

$$
\Pi(a, z)=E\left[\pi\left(a, z, \xi_{a}\right)\right]=\Psi(a)-l(z) .
$$


Riskless profit $\Psi(a)$ and expected $\operatorname{loss} l(z)$ are given by (9) and (10) substituting $\xi_{m}$ and $F_{m}$ with $\xi_{a}$ and $F_{a}$ in expected quantities expressions, where $\Lambda(z)$ are expected leftovers and $\Theta(z)$ are expected shortages.

From expressions (18) we can see that the decisions on $a$ and $z$ are made independently, unlike in the multiplicative model (see (11) and (14)). Therefore, for the additive demand model, the optimal advertising $a^{*}$ is always equal to the optimal riskless advertising $a_{\Psi}^{*}$ (see (16)), while the optimal stocking quantity $z^{*}$ corresponds to that from the multiplicative case (substituting $F_{m}$ with $F_{a}$ ), see $(12)$.

\section{Numerical examples and results for multiplicative case}

So far, we have discussed conditions for existence of a (non-zero) solution as well as theorems ensuring its uniqueness; both using the input parameters $p, c, s, v$, parameters of $d(a)$ and, especially, distribution function $F_{m}$ and $F_{a}$, respectively. In order to exemplify managerial information on the optimal decisions, we further provide some insights using the uniform distribution of the random variable.

\subsection{Uniform distribution}

Let the random variable $\xi_{m}$ be uniformly distributed, i.e., $\xi_{m} \sim U\left(A_{m}, B_{m}\right)$. Then, from (12), we get $z^{*}=A_{m}+\frac{(p+s-c)\left(B_{m}-A_{m}\right)}{p+s-v}$. Substituting $z^{*}$ into (10) we get $l\left(z^{*}\right)=\left(z^{*}-A_{m}\right) \frac{c-v}{2}=\frac{B_{m}-A_{m}}{2}(c-v) \frac{p+s-c}{p+s-v}$. Using the obtained $l\left(z^{*}\right)$, condition $p-c-l\left(z^{*}\right)>0$ from Assumption 1 converts to

$$
p-c-\frac{B_{m}-A_{m}}{2}(c-v) \frac{p+s-c}{p+s-v}>0 .
$$

Example 1 Let us consider a product with $p=15, c=10, v=8$ and $s=2$. Let $d_{0}=100, a_{\max }=150$ and $\left[A_{1}, B_{1}\right]=[0.5,1.5]$. Then $z^{*} \doteq 1.278 \Rightarrow$ $l\left(z^{*}\right) \doteq 0.778 \Rightarrow p-c-l\left(z^{*}\right) \doteq 4.222$ and so Assumption 1 is satisfied (or condition (19), alternatively).

In this section, we further consider the uniform distribution with the parameters from Example 1.

\subsubsection{Impact of demand function}

To meet Assumption 2, we choose the following parameters for the response functions given by (1)-(3) in Table 1, where we also present one example for the S-shaped function, for which Assumption 2 is not met (and so $\Pi\left(a, z^{*}\right)$ is not quasi-concave). Note that $\frac{1}{p-c-l\left(z^{*}\right)} \doteq 0.237$ and note that for functions (1)-(3) we can use conditions (from Assumption 2) given by Remark 3. 
Table 1: Numerical examples of response functions given by (1)-(3).

\begin{tabular}{|c|c|c|c|c|c|c|c|c|}
\hline Type & Indic. $\|$ & & Parameters & & $\frac{\mathrm{d} d(0)}{\mathrm{d} a}$ & Ass. 2 & $\frac{\mathrm{d} d(150)}{\mathrm{d} a}$ & Ass. 2 \\
\hline (1) & $d_{1}(a)$ & $\alpha=\frac{3}{10}$ & $\omega=20$ & & $\infty$ & $\checkmark$ & 0.180 & $\checkmark$ \\
\hline (2) & $d_{2}(a)$ & $\theta=100$ & $\delta=\frac{1}{2}$ & & 50 & $\checkmark$ & 0.027 & $\checkmark$ \\
\hline (3) & $d_{3}(a)$ & $\theta=100$ & $\theta_{l}=\frac{1}{2}$ & $\gamma=\frac{1}{2}$ & 0.249 & $\checkmark$ & 0.165 & $\checkmark$ \\
\hline (3) & $d_{4}(a)$ & $\theta=100$ & $\theta_{l}=\frac{1}{2}$ & $\gamma=\frac{1}{10}$ & 0.050 & $x$ & 0.001 & $\checkmark$ \\
\hline
\end{tabular}

See Figure 2a where we illustrate these four concrete response functions and see Figure $2 \mathrm{~b}$ for their related expected profit functions. We can see that Assumption 2 is violated for $d_{4}(a)$ (especially, the left hand derivative, see also Table 1). This simply means that the advertising costs are higher than the revenues during the initial period. Hence, advertising is not effective/profitable here. Then, even if the optimal advertising is unique for all the examples $d_{1}(a)-d_{4}(a)$, the solution of the first order condition (14) determines two critical points for example $d_{4}(a)$ and so Theorem 2 does not hold since the expected profit function is not quasi-concave.

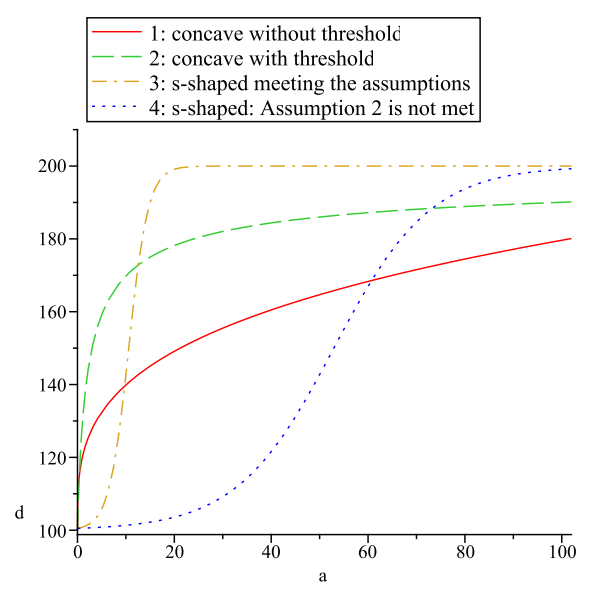

(a) Response function examples $d_{1}(a)-d_{4}(a)$.

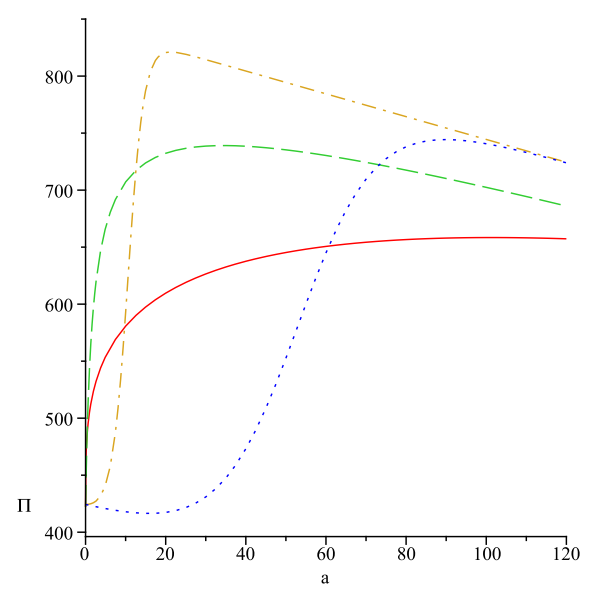

(b) Expected profit functions $\Pi\left(a, z^{*}\right)$.

Fig. 2: Examples of response functions and related expected profit functions.

In Table 2, we provide numerical results of optimal quantities for response function examples $d_{1}(a)-d_{4}(a)$. The expected profits $\Pi\left(a^{*}, x^{*}\right)$ and the related quantities can be compared for the multiplicative and riskless demand cases. Moreover, the optimal order quantities $x^{*}$ as well as expected profits can be compared with the related values of the standard NP presented by Khouja (1999). For simplicity, the optimal function values and optimal values of orig- 
inal decision variables are denoted as $a^{*}, d\left(a^{*}\right), x^{*}, \Pi\left(a^{*}, v^{*}\right)$ for considered models in Table 2 .

Table 2: Numerical results of Example 1 for the uniform distribution for two cases: multiplicative and riskless; numerical results of the equivalent NP

\begin{tabular}{|c|c|c|c|c|c|c|c|c|c|}
\hline Problem & $\|$ & Multiplic & tive NI & & $\|$ & Riskle & s NPA & & NP \\
\hline & $\| d_{1}(a)$ & $d_{2}(a)$ & $d_{3}(a)$ & $d_{4}(a)$ & $\| d_{1}(a)$ & $d_{2}(a)$ & $d_{3}(a)$ & $d_{4}(a)$ & - \\
\hline$a^{*}$ & 101.2 & 34.5 & 21.3 & 89.9 & 128.9 & 38.7 & 21.6 & 91.6 & - \\
\hline$d\left(a^{*}\right)$ & 179.9 & 183.2 & 199.5 & 197.6 & 185.9 & 184.1 & 199.6 & 198.0 & _ \\
\hline$x^{*}$ & 229.9 & 234.1 & 254.9 & 252.5 & 187.2 & 185.4 & 200.9 & 199.2 & 127.8 \\
\hline$\Pi\left(a^{*}, x^{*}\right)$ & 658.4 & 739.1 & 821.2 & 744.3 & 799.9 & 881.2 & 975.6 & 897.4 & 444.4 \\
\hline
\end{tabular}

In Figure 3a, we illustrate how parameter $\gamma$ of the S-shaped function affects the Assumption 2. If the left hand derivative of the response function is less than $1 /\left[p-c-l\left(z^{*}\right)\right]$, then the Assumption 2 is violated (left "negative" part of $d_{4}(a)$ in Figure 3a). In other words, for small $\gamma$ 's is the advertising not effective in the initial period, i.e. the expected profit is negative (Figure $2 b$ ).

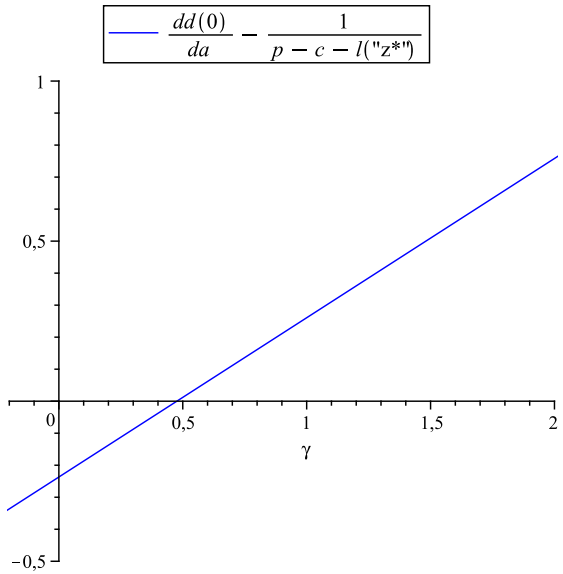

(a) Assumption 2: illustration of dependency on the S-shaped function parameter $\gamma$.

Fig. 3: Visualizations of dependencies between selected parameters and assumptions 1 and 2 .

\subsubsection{Impact of price and cost changes}

Impact on Assumption 1. Assumption 1 crucially depends on the margin $p-c$, see (19). The higher the margin the more likely the assumption will be fulfilled.

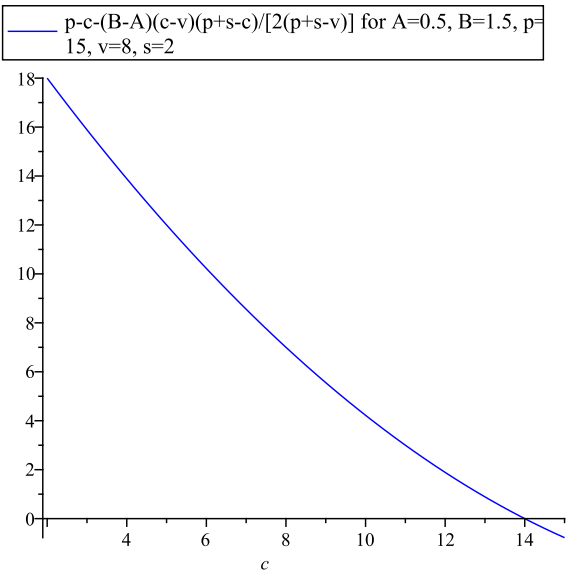

(b) Assumption 1: expected unit loss $p-c-$ $l\left(z^{*}\right)$, see (19), as function of the cost $c$. 
Therefore, we provide an insight into the impact of the margin change: in Figure 3b, we illustrate the dependency by varying $c$ (by means of (19)).

Impact on optimal advertising. Let us take the first derivative w.r.t. $a$ of the expression given by (13) and let us substitute $z^{*}$ (and $l\left(z^{*}\right)$ respectively) for the uniform distribution. Then, taking derivative w.r.t. $p$, we get

$$
\frac{\partial\left(\frac{\partial \Pi\left(a, z^{*}\right)}{\partial a}\right)}{\partial p}=\frac{\partial d(a)}{\partial a}\left[1-\frac{B_{m}-A_{m}}{2} \frac{(c-v)^{2}}{(p+s-v)^{2}}\right]>0 .
$$

Therefore, $\Pi\left(a, z^{*}\right)$ is strictly supermodular in $(a, p)$ (see Wang (2008)) and the optimal advertising is strictly increasing in selling price $p$. With the same procedure but w.r.t. $c$, we get:

$$
\frac{\partial\left(\frac{\partial \Pi\left(a, z^{*}\right)}{\partial a}\right)}{\partial c}=d(a)\left[\frac{B_{m}-A_{m}}{2} \frac{2 c-p-s-v}{p+s-v}-1\right]<0,
$$

which means that $\Pi\left(a, z^{*}\right)$ is strictly submodular in $(a, c)$ and the optimal advertising is strictly decreasing in buying cost $c$.

Therefore, any increase in the unit profit margin, i.e. range $p-c$ (see Khouja (1999)), leads to higher optimal advertising expenditure $a^{*}$.

\subsection{Discussion on the numerical results}

The numerical examples presented in Section 5 indicate how: a) various response functions as well as given various parameters settings representing different markets or market strategies influence the profit and b) profit margin changes (denoted $p-c$ ) can impact optimal advertising and expected profit.

The marketing strategy is based on the choice of advertising response function. Although such a choice can lead to increases in profit, the situation can become more complex. As we see from Figure $2 \mathrm{~b}$ and Table 2, the shape of chosen response function can significantly modify the shape of original objective function for the case with no advertising. It may change the optimal solution location from quantitative point of view, however, the shape of the objective changes also qualitatively. Therefore, the manager can design his marketing strategy, and so, the related response function in the way that will influence his operational decision making needs. Thus, although marketing decisions are not easy to perform, even in our fundamental NPA-setting, we show that even discussion of concrete explanatory examples can provide valuable insights into decision making, i.e. how to determine the optimal decisions and related quantities.

Furthermore, we have also shown how the manager can evaluate a particular situation regarding to assumptions 1 and 2. Specifically, when Assumption 1 is violated then the only one optimal strategy is to 'do nothing'. In addition, when Assumption 2 is not met then it does not necessarily mean that the advertising strategy cannot lead to increases in sales (especially for the S-shaped function), however, there is no guarantee that we will find a unique solution (advertising expenditure) of the first first order condition. 


\section{Conclusions and further research}

This study demonstrates the possibility of solving an extension of the NP model in which stocking quantity and advertising expenses are set simultaneously and in which the so-called multiplicative and additive demand cases were utilized; this in order to deal with the level of uncertainty. The major point of our research was to obtain an appropriate model for demand with an appealing and intuitive economical interpretation but one that still unified the existing concepts. Notation was adopted as used in the literature published on NPP so as to provide a simple structure of the model and its solution.

Employing this notation, an algorithm was devised to solve the problem that comprised the following steps: 1. Using input parameters, first an optimal stocking factor $z^{*}$ is determined; 2. By applying $z^{*}$ and a concrete (suitable) function $d(a)$, an optimal advertising $a^{*}$ can be expressed while $z^{*}$ and $a^{*}$ determine the optimal order quantity $x^{*}$.

As far as we are aware, two appropriate-general demand functions are identified that stem from literary sources on economics: the concave and the S-shaped response (demand) functions. For both of these, we not only solved the relevant problem, but also provided some necessary conditions to guarantee a solution that is also unique.

An important finding concerns the difference between theorems 3 and the related observation for the additive case. It was revealed that optimal advertising in the multiplicative demand case never exceeds the optimal advertising in the equivalent deterministic model while, in the additive case, it is always equal; i.e., advertising is independent of the uncertainty involved. This difference can be explained by expressions (11) and (18), which show for the additive case that the loss function is independent of the advertising amount $a$. The mentioned reference sources (Mills 1959; Karlin and Carr 1962; Petruzzi and Dada 1999) give rise to an appropriate discussion on a similar situation for NPP where the optimal price under conditions of multiplicative uncertain demand is never less than the riskless price while the optimal price under additive uncertain demand is never greater than the optimal riskless price.

We identified some original, definite functions that brought about potentially suitable advertising response functions, which subsequently produced several results and illustrations of numerical examples for uniform distribution of the random variable.

From a managerial perspective, most of the findings given herein might seem somewhat theoretical. However, a problem that simultaneously comprises advertising and pricing decisions should be of greater practical importance. Obviously, the same holds for the indication that uncertainty does not necessarily lead to greater advertising expenses. It is our intention to continue to pursue such a direction in future research, with the expectation of results that are both theoretically and practically relevant. We believe that our results add valuable managerial insight into these problems, which seems to be of higher importance in future volatile and globalised markets. The newsvendor plat- 
form is simple, and does not cover most complex practical situations. Still, the need for more research in this direction is evident.

As further challenging topics, we have identified: an extension of the advertising response functions to other S-shaped functions (e.g. Kozusko and Bourdeau 2015), its generalizing and focus on the variability of the random factor (Khouja 1999) as decision dependent randomness (Hrabec et al 2012). Moreover, the advertising decisions can be combined with other operational decisions such as pricing (Petruzzi and Dada 1999); other aspects as risk analysis (Jammernegg and Kischka 2007) or any combination of the topics (Dai and Meng 2015) are also challenging. Cost variability ideas (e.g. Novák and Popesko 2014) may also lead to new interesting results.

A particularly interesting motivating application problem has recently appeared in waste-to-energy generation. Ferdan et al (2015) discussed the problem of finding the optimal waste-to-energy plant capacity with respect to the uncertain future demand for the heat and electricity. We have found that such a problem in its reduced form can be initially modeled by the NP. Such a simplification can be very useful for the initial managerial strategical decision about the principal investment level. This decision can be further precised by utilization of more complex model. In addition, the usual interplay between strategical decision about capacity and returns under uncertain demands can be originally enriched by the impact of advertising. The advertising process is then related to the information campaign directed towards the waste producers and the pricing mechanism is linked to a so-called gate fee price for the waste processed. Hence, in this recent real-world management problem, combining strategical investment policy and operational decisions with respect to uncertain demands, we plan to use the present research results.

Acknowledgements The authors wish to thank two anonymous referees for their very helpful comments and suggestions. This work was supported by the European Regional Development Fund under the project CEBIA-Tech Instrumentation No. CZ.1.05/2.1.00/19.0376. The study was also supported by a specific research project entitled "Modern Methods of Applied Mathematics for the Use in Technical Sciences", no. FSI-S-14-2290, id. code 25053; in addition to NETME CENTRE PLUS (LO1202), which was created with financial support from the Ministry of Education, Youth and Sports under National Sustainability Programme I. Gratitude is also expressed to the Programme EEA and Norway Grants for funding via the EEA Scholarship Programme: Bilateral Scholarship Programme, for a student mobility grant, and for grant on Institutional cooperation projects nr. NF-CZ07-ICP-4-345-2016.

\section{References}

Bagozzi RP (1986) Principles of marketing management. Science Research Associates, New York.

Bass FM (1969) A new product growth for model consumer durables. Management Science 15(5):215-227

Batra J, Aaker AA, G MJ (1995) Advertising management. Prentice Hall, 5th Edition. 
Bronnenberg BJ (1998) Advertising frequency decisions in a discrete markov process under a budget constraint. Journal of Marketing Research 35:399 406

Celikbas M, Shanthikumar JR, Swaminathan JM (1999) Coordinating production quantities and demand forecast through penalty schemes. IIE Transactions 31:851-864

Dai J, Meng W (2015) A risk-averse newsvendor model under marketingdependency and price-dependency. International Journal of Production Economics 160:220-229

Dubé JP, Hitsch G, Manchanda P (2005) An empirical model of advertising dynamics. Quantitative Marketing and Economics 3(2):107-144

Dupačová J (1994) Applications of stochastic programming under incomplete information. Journal of Computational and Applied Mathematics 56:113 125

Edgeworth FY (1888) The mathematical theory of banking. Journal of the Royal Statistical Society 51:113-127

Eliashberg J, Steinberg R (1993) Marketing-production joint decision-making. Handbooks in Operations Research and Management Science 5:827-880

Ferdan T, Šomplák R, Zavíralová L, Pavlas M, Frýba L (2015) A waste-toenergy project: a complex approach towards the assessment of investment risks. Applied Thermal Engineering 89:1127-1136

Fox J, Weisberg S (2010) Nonlinear regression and nonlinear least squares in $\mathrm{R}$ : an appendix to an $\mathrm{R}$ companion to applied regression. 2nd edition.

Freeland JR (1980) Coordination strategies for production and marketing in a functionally decentralized firm. AIIE Transactions 12:126-132

Gerchak Y, Parlar M (1987) A single period inventory problem with partially controllable demand. Computers and Operations Research 14:1-9

Hanssens DM, Parsons LJ (1993) Econometric and time-series market response models. Handbooks in Operations Research and Management Science 5:409 464

Hausman WH, Montgomery DB, Roth AV (2002) Why should marketing and manufacturing work together? Some exploratory empirical results. Journal of Operations Management 20:241-257

Hrabec D, Popela P, Novotny J, Haugen K, Olstad A (2012) A note on the newsvendor problem with pricing. In: 18th International Conference on Soft Computing, MENDEL 2012, pp 410-415

Jammernegg W, Kischka P (2007) Risk-averse and risk-taking newsvendors: a conditional expected value approach. Review of Managerial Science 1:93-110

Jones JP (1995) When ads work-new proof the advertising triggers sales. Lexington Books, New York.

Karlin S, Carr SR (1962) Prices and optimal inventory policy. Studies in Applied Probability and Management Science pp 299-336

Khouja M (1999) The single-period (news-vendor) problem: literature review and suggestions for the future research. Omega 27:537-553

Khouja M, Robbins SS (2003) Linking advertising and quantity decisions in the single-period inventory model. International Journal of Production Eco- 
nomics 86:93-105

Kotler P, Armstrong G (2013) Principles of marketing. Prentice Hall, 15th edition.

Kozusko F, Bourdeau M (2015) The trans-gompertz function: an alternative to the logistic growth function with faster growth. Acta Biotheoretica $63(4): 397-405$

Lee CM, Hsu SL (2011) The effect of advertising on distribution-free newsboy problem. International Journal of Production Economics 129:217-224

Leitch RA (1974) Marketing strategy and optimal production schedule. Management Science 21:302-312

Malhotra MK, Sharma S (2002) Spanning the continuum between marketing and operations. Journal of Operations Management 20:209-219

Mills ES (1959) Uncertainty and price theory. The Quarterly Journal of Economics $73: 116-130$

Monahan G, Petruzzi N, Zhao W (2002) The dynamic pricing problem from a newsvendor's perspective. Manufacturing and Service Operations Management $6(1): 73-91$

Mooradian T, Matzler K, Ring L (2011) Strategic marketing. Prentice Hall.

Novák P, Popesko B (2014) Cost variability and cost behaviour in manufacturing enterprises. Economics and Sociology 7(4):89-103

Petruzzi NC, Dada M (1999) Pricing and the newsvendor problem: a review with extensions. Operations Research 47(2):183-194

Phillips R (2005) Pricing and revenue optimization. Stanford: Stanford Business Books.

Porteus EL (1990) Stochastic inventory theory. Handbooks in Operations Research and Management Science 2:605-652

Ruszczynski A, Shapiro A (2003) Stochastic programming. Handbooks in Operations Research and Management Science 10. North-Holland.

Shapiro A, Dentcheva D, Ruszczynski A (2009) Lectures on stochastic programming: modeling and theory. SIAM.

Shi X, Zhang S (2014) Trade credit: the interaction of financing, marketing, operations, and risk behavior. Review of Managerial Science 8:225-248

Silver EA, Peterson R (1985) Decision systems for inventory management and production planning. John Wiley, New York.

Simon JA, Arndt J (1980) The shape of advertising response function. Journal of Advertising Research 20(4):11-28

Wang T (2008) The newsvendor problem with advertising. In: IEEE International Conference on Service Operations and Logistics, and Informatics (IEEE/SOLI), Shanghai, China, pp 886-889

Wang T (2009) Joint advertising and ordering strategies for perishable product with emergency ordering. In: Control and decision conference (CCDC), Shanghai, China, pp 2673-2678

Wang T (2011) Joint advertising and ordering strategies for perishable product under general demand. In: Third International Conference on Artificial Intelligence and Computational Intelligence, AICI, Taiyuan, China, pp 525532 
Wang T, Hu Q (2011) Coordination of supply chain with advertise-and-pricesetting newsvendor. In: Control and decision conference (CCDC), Shenyang, China, pp 885-890

Whitin TM (1955) Inventory control and price theory. Management Science $2: 61-68$

Xinsheng X, Zhiqing M, Rui S, Min J, Ping J (2015) Optimal decisions for the loss-averse newsvendor problem under CVaR. International Journal of Production Economics 164:146-159

Yao L, Chen YF, Yan H (2006) The newsvendor problem with pricing: extensions. International Journal of Management Science and Engineering Management $1(1): 3-16$

Zhan RL, Shen MZ (2005) Newsvendor problem with pricing: properties, algorithms, and simulations. In: Proceedings of the 2005 Winter Simulation Conference, University of Florida, pp 1743-1748 